\title{
Acute effects of elevated NEFA on vascular function: a comparison of SFA and MUFA
}

\author{
Katie J. Newens, Abby K. Thompson, Kim G. Jackson, John Wright and Christine M. Williams* \\ Hugh Sinclair Unit of Human Nutrition, Department of Food and Nutritional Sciences, University of Reading, \\ Reading RGG 6AP, UK
}

(Received 25 May 2010 - Revised 24 September 2010 - Accepted 1 November 2010 - First published online 16 December 2010)

\section{Abstract}

There is emerging evidence to show that high levels of NEFA contribute to endothelial dysfunction and impaired insulin sensitivity. However, the impact of NEFA composition remains unclear. A total of ten healthy men consumed test drinks containing $50 \mathrm{~g}$ of palm stearin (rich in SFA) or high-oleic sunflower oil (rich in MUFA) on separate occasions; a third day included no fat as a control. The fats were emulsified into chocolate drinks and given as a bolus (approximately $10 \mathrm{~g}$ fat) at baseline followed by smaller amounts (approximately $3 \mathrm{~g}$ fat) every $30 \mathrm{~min}$ throughout the $6 \mathrm{~h}$ study day. An intravenous heparin infusion was initiated $2 \mathrm{~h}$ after the bolus, which resulted in a three- to fourfold increase in circulating NEFA level from baseline. Mean arterial stiffness as measured by digital volume pulse was higher during the consumption of SFA $(P<0.001)$ but not MUFA $(P=0.089)$ compared with the control. Overall insulin and gastric inhibitory peptide response was greater during the consumption of both fats compared with the control $(P<0 \cdot 001)$; there was a second insulin peak in response to MUFA unlike SFA. Consumption of SFA resulted in higher levels of soluble intercellular adhesion molecule-1 (sI-CAM) at $330 \mathrm{~min}$ than that of MUFA or control $(P \leq 0.048)$. There was no effect of the test drinks on glucose, total nitrite, plasminogen activator inhibitor-1 or endothelin-1 concentrations. The present study indicates a potential negative impact of elevated NEFA derived from the consumption of SFA on arterial stiffness and sI-CAM levels. More studies are needed to fully investigate the impact of NEFA composition on risk factors for CVD.

Key words: NEFA: Incretin: Insulin: Digital volume pulse

Insulin-resistant states such as obesity and type 2 diabetes (T2D) are characterised by metabolic anomalies such as elevated plasma NEFA levels. Epidemiological studies have shown that elevated plasma NEFA are associated with a greater risk of developing impaired glucose tolerance ${ }^{(1)}$ and T2 ${ }^{(2)}$. Experimental elevation of plasma NEFA using intravenous infusion of lipid and heparin also results in impaired insulin-stimulated glucose uptake in skeletal muscle ${ }^{(3)}$ and endothelial dysfunction $^{(4)}$, both of which are key features of T2D and obesity ${ }^{(5,6)}$. There is mounting evidence to suggest that the saturation of dietary fat can influence endothelial function; for example, consumption of SFA during weightloss regimens has been shown to be negatively associated with flow-mediated dilatation $(F M D)^{(7)}$. Levels of SFA in serum have also been shown to be inversely correlated with endothelial-dependent forearm blood flow ${ }^{(8,9)}$. Findings from chronic dietary studies comparing diets rich in SFA or MUFA are mixed. Some short-term studies have found that a SFA-rich diet impairs vascular reactivity compared with a
MUFA-rich diet ${ }^{(10)}$ or a diet rich in both MUFA and PUFA ${ }^{(11)}$ However, an 8-month supplementation trial found no difference in endothelial-dependent or -independent vasodilation measures between MUFA- or SFA-based oils ${ }^{(12)}$. Similarly, there are contrasting findings from postprandial studies comparing high-fat meals rich in either SFA or MUFA, with some studies finding no differential effect on FMD $^{(13,14)}$ and one study reporting a negative impact of a MUFA-rich meal on FMD ${ }^{(15)}$.

Dietary fat composition may affect endothelial function by modulating insulin sensitivity since the endothelium is an insulin-dependent tissue, and impaired endothelial function is a key feature of T2D and other insulin-resistant states. Although the impact of dietary fatty acid composition on insulin sensitivity remains equivocal ${ }^{(16)}$, one large randomised controlled trial found a decrease in insulin sensitivity after substitution of monounsaturated fat for saturated fat over a 12-week period when total fat consumed constituted less than $37 \%$ energy ${ }^{(17)}$. Furthermore, the Atherosclerosis Risk in Community Study found that the percentage of SFA in

Abbreviations: ET-1, endothelin-1; FMD, flow-mediated dilatation; GIP, gastric inhibitory peptide; GLP-1, glucagon-like peptide-1; NO , total nitrite; PAI-1, plasminogen activator inhibitor-1; $\mathrm{SI}_{\mathrm{DVP}}$, stiffness index derived from digital volume pulse; sI-CAM, soluble intercellular adhesion molecule-1; T2D, type 2 diabetes. 
plasma cholesteryl esters and phospholipids was positively associated with the development of diabetes ${ }^{(18)}$.

Mechanisms that might mediate the variable effects of dietary fat on insulin sensitivity and endothelial function include potentially direct effects of fatty acids on insulin signalling pathways in endothelial cells, as has been described for skeletal muscle ${ }^{(3)}$. In addition, there are potentially indirect effects of different fatty acids on insulin secretion. The possibility that this might be mediated via an effect on the secretion of incretin hormones (gastric inhibitory peptide (GIP) and glucagon-like peptide-1 (GLP-1)) is of particular interest given the emerging therapeutic role of GLP-1, in particular in the treatment of T2D. Indeed, infusion of GLP-1 has been shown to cause a marked increase in FMD in T2D patients with stable coronary artery disease ${ }^{(19)}$. Whether such benefits in incretin secretion might be achieved by dietary changes is unclear, but postprandial studies have indicated that consumption of MUFA-rich meals induces a higher incretin response than consumption of SFA-rich meals ${ }^{(20,21)}$. Furthermore, Southern European subjects whose habitual diets were rich in MUFA have been shown to have fasting levels of insulin twofold higher and fasting levels of GIP over fourfold higher than a matched group of Northern European subjects ${ }^{(22)}$.

In addition to effects on insulin secretion, fatty acids may have a direct impact on the insulin signalling pathway, influencing the balance between vasodilator and vasoconstrictor responses. Insulin signalling through the phosphoinositide-3 kinase pathway regulates the production of the vasodilator $\mathrm{NO}$, an important regulator of vascular tone ${ }^{(23)}$. However, an alternative pathway of insulin signalling, the mitogen-activated protein kinase pathway, can lead to the generation of the vasoconstrictor endothelin-1 (ET-1), as well as cellular adhesion molecules such as soluble intercellular adhesion molecule-1 (sI-CAM) and plasminogen activator inhibitor-1 (PAI-1). Evidence from cell culture studies suggests that different fatty acids may affect the balance of these two pathways, with particularly adverse effects of SFA, which may have significance for impaired endothelial function and atherosclerosis ${ }^{(24,25)}$.

Previous experimental studies of acute NEFA elevation have been unable to study variable effects of fatty acid composition since they have mostly used intravenous lipid emulsions such as Intralipid, which are rich in $n$ - 6 PUFA. The present study aimed to investigate the effects of acute elevation of plasma NEFA of different composition using a relatively new protocol developed by Beysen et al. ${ }^{(26)}$, which involves frequent ingestion of fat administered orally rather than by intravenous infusion. We have examined the effect of elevating NEFA derived from consumption of SFA- and MUFA-rich drinks on the levels of insulin and incretin hormones, as well as on circulating measures of endothelial function (total nitrite $\left(\mathrm{NO}_{x}\right)$, sI-CAM, ET-1 and PAI-1). In addition, we used digital volume pulse to assess arterial stiffness as a further marker of endothelial function.

\section{Experimental methods}

The present study was conducted according to the guidelines laid down in the Declaration of Helsinki, and all procedures involving human subjects were approved by the University of Reading Ethics Committee. Written informed consent was obtained from all subjects. The study was conducted in the Hugh Sinclair Unit of Human Nutrition at the University of Reading.

The method of elevating NEFA of specific types is based on that by Beysen et al. ${ }^{(27)}$. A drink mixture was prepared comprising $50 \mathrm{~g}$ of either palm stearin (SFA; 59\% 16:0, 28\% $18: 1 n-9,6 \% 18: 2 n-6,5 \% 18: 0)$ or high-oleic sunflower oil (MUFA; 81\% 18:1n-9, 10\% 18:2n-6, 4\% 16:0, 3\% 18:0), $30 \mathrm{~g}$ skimmed milk powder (Premier International Foods Limited, Birmingham, UK), $15 \mathrm{~g}$ chocolate powder (The Spanish Chocolate Company Limited, Leicester, UK) and $0.5 \mathrm{~g}$ monoacylglycerol emulsifier (Danisco, Copenhagen, Denmark). Water was added to achieve a final weight of $320 \mathrm{~g}$, and the mixture was blended for several minutes to ensure emulsification. The test drinks were identical in protein $(11.5 \mathrm{~g})$ and carbohydrate $(28.1 \mathrm{~g})$ content. The drinks were divided into a main test drink of $62 \mathrm{~g}$ (approximately $10 \mathrm{~g}$ fat, $5.4 \mathrm{~g}$ carbohydrate and $2.2 \mathrm{~g}$ protein) and twelve smaller drinks of $21 \mathrm{~g}$ (approximately $3 \mathrm{~g}$ fat, $1.8 \mathrm{~g}$ carbohydrate and $0.75 \mathrm{~g}$ protein). Control drinks were prepared using the same method but excluding the oils. All drinks were served warm to enhance palatability.

Healthy men ( $n$ 10; Table 1 ) were recruited to take part in three study days. All subjects were non-smokers of normal body weight and were not suffering from hyperlipidaemia or any endocrine disorder likely to affect lipid metabolism; all showed normal lipid and standard clinical biochemistry values on screening. Subjects attended the Unit on three occasions separated by at least 1 week and were randomly assigned to one of the test drinks on each day. For $24 \mathrm{~h}$ before each study day, subjects were asked to refrain from strenuous exercise and alcohol and were supplied with a low-fat ready meal to be consumed in the evening. On each study day, subjects arrived after an overnight fast, and an intravenous cannula was placed at the wrist of the non-dominant arm. The main test drink was consumed at $0 \mathrm{~min}$, with the smaller drinks given every $30 \mathrm{~min}$ for a further $6 \mathrm{~h}$. At $120 \mathrm{~min}$, a second cannula was inserted into the antecubital vein in the non-dominant arm for the infusion of heparin. A bolus of heparin ( $500 \mathrm{IU}$ ) was administered, followed by a

Table 1. Baseline characteristics of the ten male subjects (Mean values and standard deviations)

\begin{tabular}{lrr}
\hline & Mean & SD \\
\hline Age (years) & 24.8 & 7.2 \\
Ht (m) & 1.8 & 0.1 \\
Wt (kg) & 74.2 & 10.2 \\
BMI (kg/m ${ }^{2}$ ) & 22.5 & 2.4 \\
Waist circumference (cm) & 84.1 & 5.4 \\
BP (mmHg) & & \\
Systolic & 128 & 10 \\
Diastolic & 74 & 5 \\
Fasting total cholesterol (mmol/l) & 4.1 & 0.9 \\
Fasting glucose (mmol/l) & 5.5 & 0.6 \\
Fasting TAG (mmol/l) & 0.9 & 0.3 \\
\hline
\end{tabular}

$\mathrm{BP}$, blood pressure. 
continuous infusion of heparin $(0.4 \mathrm{IU} / \mathrm{kg}$ body weight per min) for the rest of the study day.

Stiffness index derived from digital volume pulse ( $\left.\mathrm{SI}_{\mathrm{DVP}}\right)$ was measured four times during each study day using digital volume pulse with a PulseTrace PCA 2 (Micro Medical Limited, Kent, UK). $\mathrm{SI}_{\mathrm{DVP}}$ has been shown to be positively correlated with classical CVD risk factors such as age, waist:hip ratio, blood pressure and carotid intima-media thickness ${ }^{(28)}$. The PulseTrace equipment calculates $\mathrm{SI}_{\mathrm{DVP}}$ by recording three consecutive waveforms through the index finger pulse; $\mathrm{SI}_{\mathrm{DVP}}$ is calculated by dividing the subject's height by the difference in time between the systolic-derived peak and the diastolicderived peak of the waveform. In addition to the replicates performed by PulseTrace, this technique was performed three times, giving an average of nine readings at each time point.

Blood samples were collected every $30 \mathrm{~min}$ into $\mathrm{K}_{3}$ EDTA or serum tubes and centrifuged at $4^{\circ} \mathrm{C}$ within $30 \mathrm{~min}$ of collection. For analysis of GIP, $10 \mu \mathrm{l}$ of dipeptidyl peptidase IV inhibitor (Millipore Corporation, Watford, Herts, UK) per ml of blood were added at the time of collection to protect against proteolysis. Samples were stored immediately at $-20^{\circ} \mathrm{C}$ until analysis. Serum NEFA were quantified using an automated clinical chemistry analyser (ILAB 600) with kits supplied by Alpha Laboratories (Eastleigh, Hants, UK). Instrumentation Laboratories (Warrington, Ches, UK) supplied kits for the analysis of serum TAG, glucose and total cholesterol. Serum insulin was measured using a dissociation-enhanced lanthanide fluorescent immunoassay kit (Perkin Elmer Limited, Beaconsfield, Bucks, UK). Plasma GIP and serum C-peptide were measured using commercially available colorimetric ELISA kits (Millipore Corporation, Watford, Herts, UK) as were serum sI-CAM, plasma PAI-I and ET-1 (R\&D Systems Europe Limited, Abingdon, Oxon, UK). Total serum nitrites $\left(\mathrm{NO}_{x}\right)$ were measured using a NO quantification kit (Actif Motif, Rixensart, Belgium).

In four subjects, fatty acid composition analysis of NEFA and TAG was performed on baseline fasted samples and those collected between 300 and 360 min following the start of the oral fat load. Lipids were extracted from $800 \mu \mathrm{l}$ serum using chloroform-methanol (2:1, by vol.) containing butylated hydroxytoluene $(50 \mathrm{mg} / \mathrm{ml})$ before being applied to a solidphase extraction cartridge (Varian, Oxford, Oxon, UK) to isolate the NEFA and TAG fractions. These fractions were then saponified and methylated in methanol containing $2 \%$ (v/v) $\mathrm{H}_{2} \mathrm{SO}_{4}$ at $70^{\circ} \mathrm{C}$ for $1 \mathrm{~h}$. Fatty acid methyl esters were recovered by extraction into hexane and analysed in a gas chromatograph as described previously ${ }^{(29)}$. Fatty acid methyl esters were identified by comparison of retention times against a known standard, Supelco 37 component fatty acid methyl esters mix (Supelco, Dorset, UK).

\section{Statistical analysis}

All statistical analyses were performed using SPSS software (version 15.0; SPSS, Inc., Chicago, IL, USA). Data were tested for normality using the Shapiro-Wilk test and log-transformed if necessary. Analytes measured at least four times a day
(NEFA, TAG, glucose, insulin, C-peptide and GIP) were compared using repeated-measures ANOVA. This approach was also used to compare the fatty acid composition of NEFA and TAG between the study days. Analytes measured only at 0 and $330 \mathrm{~min}$ (sI-CAM, NO ${ }_{x}$, ET-1 and PAI-1) were analysed with repeated-measures ANCOVA using $330 \mathrm{~min}$ as the dependent variable and $0 \mathrm{~min}$ as a covariate. In both cases, a Bonferroni correction was applied to control for multiple comparisons. Values of $P \leq 0.05$ were considered as significant.

\section{Results}

Baseline subject characteristics are shown in Table 1. There were no significant differences in any baseline measures between the test drinks.

\section{Serum NEFA, TAG and glucose}

There was an initial decline in NEFA after consumption of the bolus oral load followed by a sharp increase at $120 \mathrm{~min}$ following initiation of heparin infusion. The oral fat-heparin protocol resulted in a threefold elevation of serum NEFA following consumption of both fats compared with that of the control (Fig. 1). Repeated-measures ANOVA revealed a significant effect of test drink and time $(P<0.001)$; both MUFA- and SFA-rich drinks $(P<0 \cdot 001)$ elicited a higher NEFA response than the control, with no significant difference between the two fats $(P=0 \cdot 193)$.

The fatty acid composition of NEFA and TAG at baseline $(t=0 \mathrm{~min})$ and after consumption of the control, SFA and MUFA-rich drinks $(t=300-360 \mathrm{~min})$ is shown in Table 2. A significant effect of test drink was found for palmitic acid (NEFA and TAG, $P<0.001$ ), stearic acid (NEFA only, $P=0 \cdot 001$ ), oleic acid (NEFA and TAG, $P \leq 0 \cdot 001)$ and linoleic acid (NEFA $P=0.001$ and TAG $P=0.020$ ). There was an approximately 1.5 -fold increase in the palmitic acid content of both NEFA and TAG following consumption of the SFA test drinks. Consumption of the MUFA test drinks led to an approximately $2 \cdot 5$-fold increase of oleic acid in NEFA and an approximately $1 \cdot 7$-fold increase in TAG, which was accompanied by significant decreases in palmitic acid. Consumption of the control drinks did not affect proportions of any of the fatty acids from baseline in either NEFA or TAG.

A significant effect of test drink, time and test drink $\times$ time interaction (all $P<0 \cdot 001$ ) was found for serum TAG (Fig. 1(b)) Consumption of both MUFA- and SFA-rich drinks $(P<0.001)$ was followed by a rise in serum TAG with no rise following consumption of the control drink. There was an initial decline in serum TAG following the administration of heparin at $120 \mathrm{~min}$, but thereafter, values remained constant. There was no significant difference in the serum TAG profiles for the two test fats $(P=1 \cdot 000)$.

Serum glucose levels initially increased at $30 \mathrm{~min}$ following the bolus oral load on all three drinks and then declined to below-fasting levels until 240 min when there was a steady increase, most markedly for the two fat test drinks following heparin infusion (Fig. 2(c)). There was a significant effect of time $(P<0 \cdot 001)$ but not of test drink $(P=0 \cdot 118)$. 

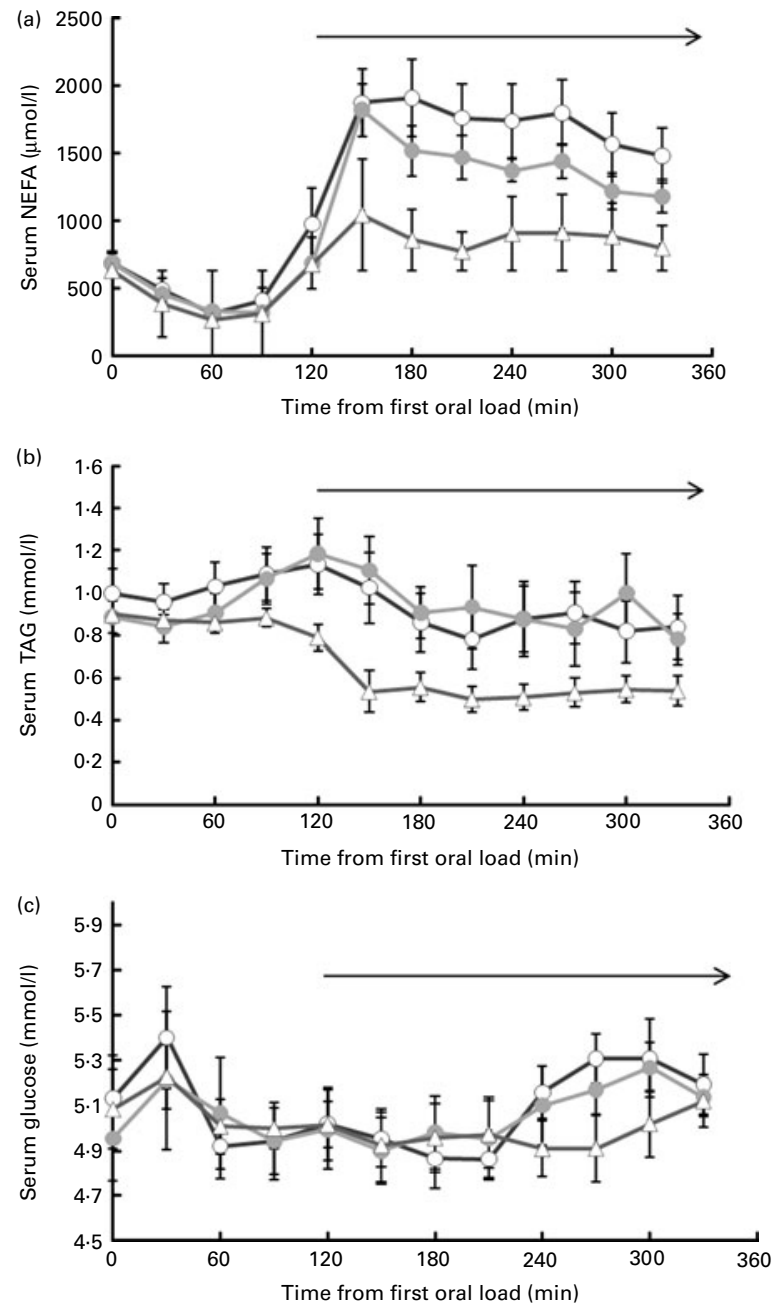

Fig. 1. Serum (a) NEFA, (b) TAG and (c) glucose (note the broken scale of the $y$-axis) during the consumption of SFA (O), MUFA (o) or control, no fat $(\triangle)$. Values are means of ten subjects, with their standard errors represented by vertical bars; $\longrightarrow$ indicates administration of heparin. For serum NEFA and TAG, repeated-measures ANOVA revealed a significant effect of test drink $(P<0.001)$, time $(P<0.001)$ and test drink $\times$ time interaction (NEFA $P=0.031$, TAG $P<0.001)$. For glucose, there was a significant effect of time only $(P<0.001)$.

\section{Serum insulin, C-peptide and gastric inhibitory peptide response}

Serum insulin levels increased following the bolus oral load of both fats, reaching a peak at $60 \mathrm{~min}$ (Fig. 2(a)). There was no insulin response to consumption of the control drink. Following administration of heparin, there was a sharp increase in insulin for the MUFA-rich drink at $150 \mathrm{~min}$ (95\% CI 43.3 , $65.3 \mathrm{pmol} / \mathrm{l})$, which was significantly different from the control at this time point $(95 \%$ CI $14.4,36.9 \mathrm{pmol} / \mathrm{l})$. In contrast, there was no rise in serum insulin at $150 \mathrm{~min}$ for the SFA-rich drink (95\% CI $27 \cdot 1,49 \mathrm{pmol} / \mathrm{l})$. There was a significant effect of test drink, time and test drink $\times$ time interaction for overall insulin response $(P<0 \cdot 001)$, with both SFA- and MUFA-rich drinks eliciting a higher insulin response than the control drink $(P<0 \cdot 001)$; however, there was no significant difference

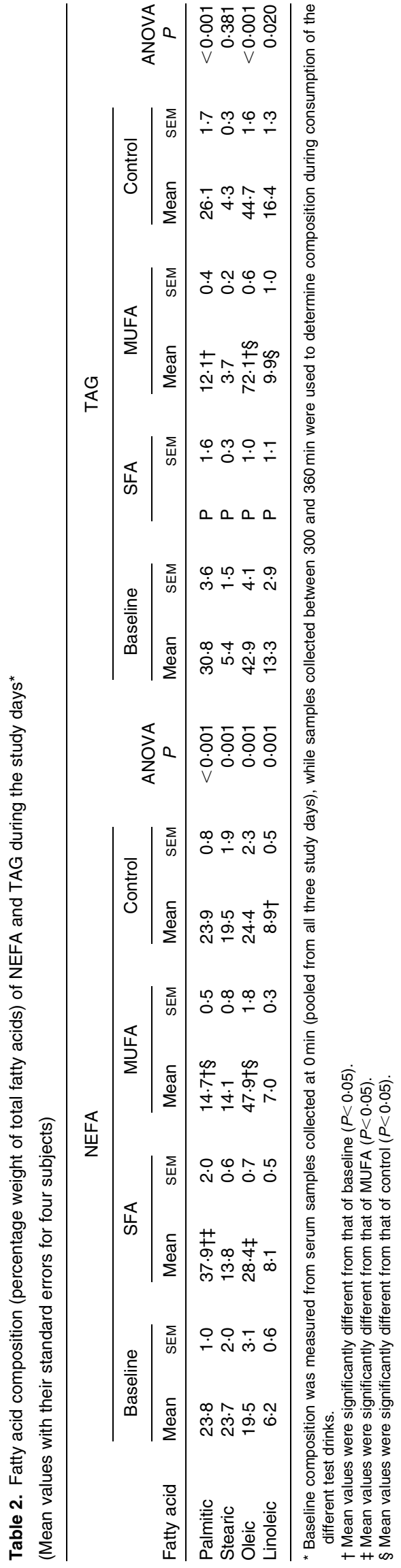



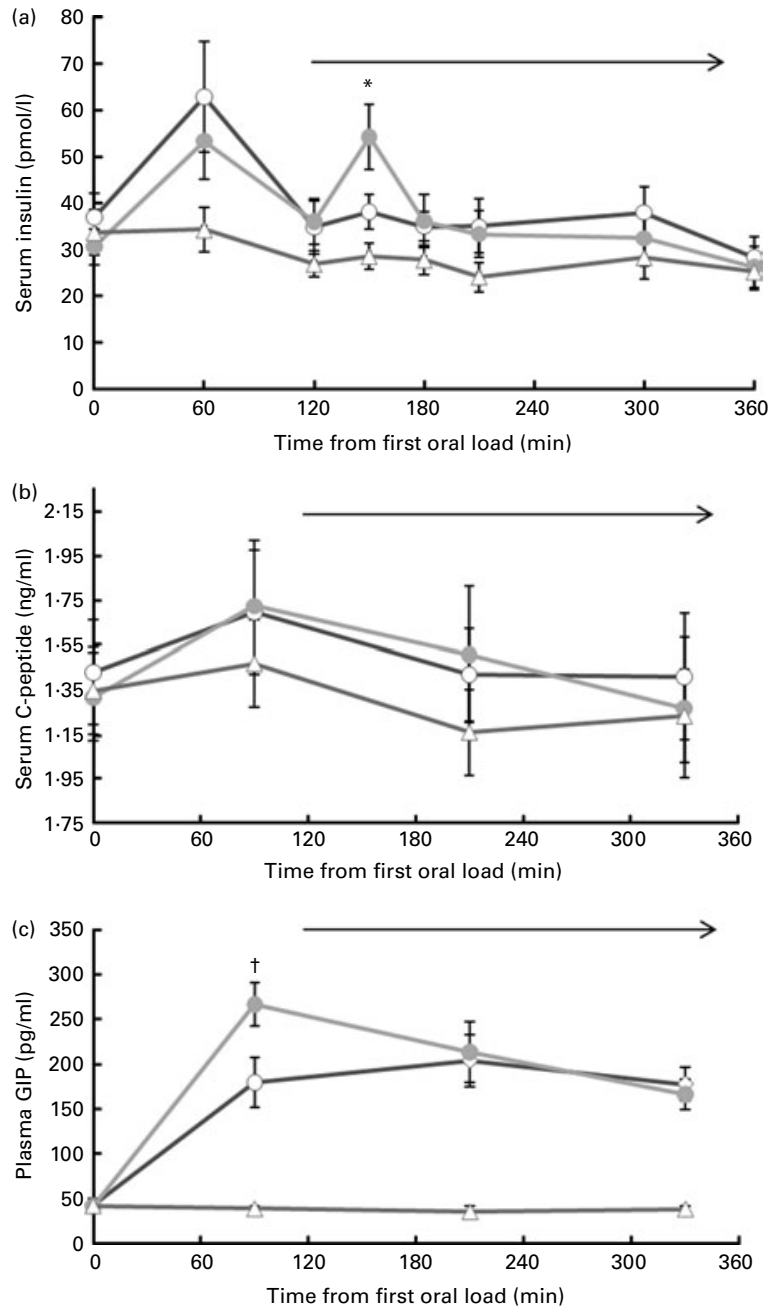

Fig. 2. Serum (a) insulin, (b) C-peptide (note the broken scale of the $y$-axis) and (c) gastric inhibitory peptide (GIP) during the consumption of SFA (O), MUFA $(\bullet)$ or control, no fat $(\triangle)$. Values are means of ten, seven and nine subjects, respectively, with standard errors represented by vertical bars; $\longrightarrow$ indicates administration of heparin. ${ }^{*}$ Denotes a significantly higher response for MUFA than control; †denotes a significantly higher response for MUFA than for SFA $(P<0.05)$. For insulin and GIP, repeated-measures ANOVA revealed a significant effect of test drink, time and test drink $\times$ time interaction $(P<0.001)$. A significant effect of test drink $(P=0.010)$ and time $(P<0.001)$ was observed for $C$-peptide.

between the fats $(P=1 \cdot 000)$. For $\mathrm{C}$-peptide, there was a significant effect of test drink $(P=0 \cdot 010)$ and time $(P<0 \cdot 001)$ (Fig. 2(b)). Compared with the control drink, the response was significantly higher for the SFA-rich drink $(P=0.013)$, with also a strong trend for MUFA values to be higher than the control $(P=0.056)$, but there was no difference between the fats $(P=1 \cdot 000)$.

There was a marked increase in plasma GIP following the consumption of both fat-containing drinks, which was sustained for the duration of the study day (Fig. 2(c)) Repeated-measures ANOVA revealed a significant effect of test drink, time and test drink $\times$ time interaction $(P<0.001)$; both fats induced a higher response than the control $(P<0 \cdot 001)$. Despite no statistically significant overall difference between the fats $(P=0.097)$, MUFA induced a significantly higher early response at $90 \mathrm{~min}$ (95\% CI 232 $302 \mathrm{pg} / \mathrm{ml})$ than SFA (95\% CI 145, $215 \mathrm{pg} / \mathrm{ml})$.

\section{Markers of endothelial function}

Despite the high inter-subject variation in $\mathrm{SI}_{\mathrm{DVP}}$ during the study days (Fig. 3), there was a clear increase in this measure during the consumption of the SFA-rich drink, which peaked at $210 \mathrm{~min}$. In the case of the MUFA-rich drink, values declined towards $210 \mathrm{~min}$, increasing slightly at $330 \mathrm{~min}$. Because of varied baselines between the study days, $\mathrm{SI}_{\mathrm{DVP}}$ was analysed by a repeated-measures ANCOVA using mean $\mathrm{SI}_{\mathrm{DVP}}$ (90-330 $\mathrm{min})$ as the dependent variable; this revealed a significant effect of test drink $(P=0 \cdot 001)$. $\mathrm{SI}_{\mathrm{DVP}}$ was higher during the consumption of the SFA-rich drink than during the consumption of the control drink $(P<0.001)$, but there was no statistical significance between the MUFA-rich and control drinks $(P=0.089)$ or the SFA-rich and MUFA drinks $(P=0 \cdot 128)$.

Table 3 shows the effect of test drinks on circulating markers of endothelial function. There was a significant effect of test drink on sI-CAM levels $(P=0 \cdot 014)$. Pairwise analysis showed that levels of sI-CAM were significantly higher during the consumption of the SFA-rich drink than during the consumption of either the MUFA-rich $(P=0 \cdot 048)$ or the control $(P=0.021)$ drink, while there was no difference between the response to MUFA and the control $(P=1 \cdot 000)$. PAI- 1 and $\mathrm{NO}_{x}$ decreased during the study days, while ET-1 increased $(P<0 \cdot 001)$, but there was no effect of test drink compared with the control for any of these parameters.

\section{Discussion}

The present study demonstrates that acute elevation of serum NEFA by the consumption of a SFA-rich drink leads to an increase in arterial stiffness and levels of sI-CAM in healthy young men. These effects were not found following ingestion of a MUFA-rich drink, suggesting that the composition of fat consumed is an important factor in determining the impact of elevated NEFA on both vascular function and endothelial inflammation. The present protocol resulted in a three- to

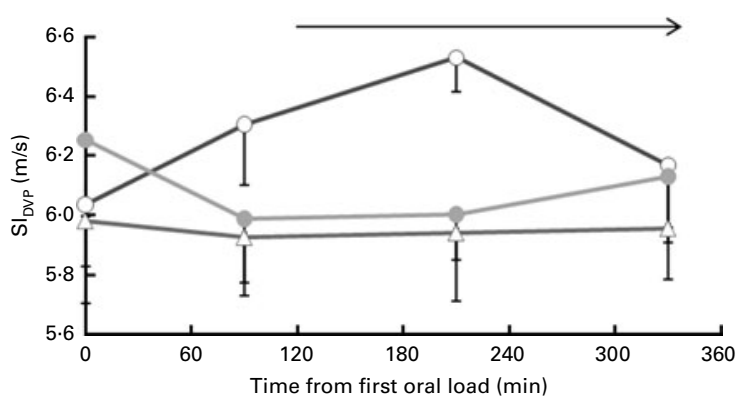

Fig. 3. Arterial stiffness index during the consumption of SFA (O), MUFA (o) or control, no fat $(\triangle)$ (note the broken scale of the $y$-axis). Values are means of eight subjects, with their standard errors represented by vertical bars; $\longrightarrow$ indicates administration of heparin. Repeated-measures ANCOVA of mean stiffness index derived from digital volume pulse $(90-330 \mathrm{~min})$ revealed a significant effect of test drink $(P=0.001)$. 
Table 3. Circulating markers of endothelial function during the study days

(Mean values with their standard errors, $n$ 10)

\begin{tabular}{|c|c|c|c|c|c|c|c|c|c|c|c|c|c|}
\hline & \multicolumn{4}{|c|}{ SFA } & \multicolumn{4}{|c|}{ MUFA } & \multicolumn{4}{|c|}{ Control } & \multirow{3}{*}{$\begin{array}{c}\text { ANCOVA } \\
P\end{array}$} \\
\hline & \multicolumn{2}{|c|}{$0 \mathrm{~min}$} & \multicolumn{2}{|c|}{$330 \mathrm{~min}$} & \multicolumn{2}{|c|}{$0 \min$} & \multicolumn{2}{|c|}{$330 \mathrm{~min}$} & \multicolumn{2}{|c|}{$0 \min$} & \multicolumn{2}{|c|}{$330 \mathrm{~min}$} & \\
\hline & Mean & SEM & Mean & SEM & Mean & SEM & Mean & SEM & Mean & SEM & Mean & SEM & \\
\hline sl-CAM (ng/ml) & 178 & 18 & $184^{*}$ & 20 & 174 & 16 & $168 \dagger$ & 16 & 168 & 17 & 166 & 17 & 0.014 \\
\hline PAl-1 (ng/ml) & 4.7 & 0.9 & 2.6 & 0.56 & 3.4 & 0.58 & $2 \cdot 2$ & 0.51 & 4.5 & 0.97 & 3.1 & 0.57 & 0.346 \\
\hline ET-1 (ng/ml) & $1 \cdot 16$ & 0.06 & 1.24 & 0.07 & 1.22 & 0.05 & 1.24 & 0.07 & 1.20 & 0.08 & $1 \cdot 20$ & 0.04 & 0.815 \\
\hline $\mathrm{NO}_{x}(\mu \mathrm{M})$ & $13 \cdot 4$ & $2 \cdot 1$ & 9.2 & 1.3 & $10 \cdot 9$ & 1.4 & 8.7 & $1 \cdot 1$ & $11 \cdot 2$ & 1.4 & $7 \cdot 1$ & $1 \cdot 1$ & 0.127 \\
\hline
\end{tabular}

sI-CAM, soluble intercellular cell adhesion molecule-1; PAI-1, plasminogen activator inhibitor-1; ET-1, endothelin-1; $\mathrm{NO}_{x}$, total nitrites

* Mean value was significantly different from that of control $(P<0.05)$.

$\dagger$ Mean value was significantly different from that of SFA $(P<0.05)$.

fourfold increase in NEFA from baseline in our healthy subjects, achieving levels observed in insulin-resistant states such as obesity and T2D. A subset analysis of fatty acid composition of NEFA and TAG also confirmed that the protocol significantly increased the proportions of palmitic and oleic acids during the SFA and MUFA study days, respectively.

The present study confirms the findings of published lipid infusion studies, which have shown that experimental acute elevation of NEFA impairs vascular function ${ }^{(4,30-36)}$; this is also reflected in postprandial studies, which have shown similar adverse effects of all types of high-fat meals ${ }^{(37)}$. However, unlike the findings of lipid infusion studies, which have analysed markers of vascular function and inflammation in response to elevating NEFA rich in $n-6$ PUFA, the protocol adopted in the present study has enabled a comparison of the acute effects of SFA and MUFA. As such, there are no previous studies to directly compare the present results, although several postprandial studies have compared the effect of mixed meals rich in SFA or MUFA on vascular function, with variable results. Comparison of outcomes from different postprandial studies is complicated by virtue of differences in the amount and source of the fats used in the oral fat load. For example, shea butter is rich in stearic acid and has been shown to have varying absorption rates following acute ingestion, which would confound data from this type of study ${ }^{(38)}$. In addition, the amount and type of carbohydrate in test meals in different studies is also variable which, through differing effects on insulin secretion, would introduce additional variables other than the fat type. Raitakari et al. ${ }^{(14)}$ reported that FMD did not change after a cooked breakfast rich in SFA or MUFA. Rueda-Clausen et $a l^{(13)}$ also found a similar reduction in FMD after consumption of potato soup containing either olive oil or palm oil. In contrast, Berry et al. ${ }^{(15)}$ found a significant reduction in FMD after a meal of muffins and a milkshake enriched in high-oleic sunflower oil, which was not found when the meal was enriched with shea butter. However, in this latter study, consumption of the MUFA-rich meal led to significantly higher circulating TAG levels than consumption of the SFA-rich meal. This could affect vascular function as the extent of postprandial lipaemia has been shown to be negatively correlated with FMD in healthy subjects consuming high-fat meals ${ }^{(39,40)}$. In the present study, the oils used for the SFA fat load were chosen to reflect typical UK fatty acid intakes, and, importantly, no difference was recorded between circulating TAG levels during consumption of either the SFA- or the MUFA-rich fat loads, suggesting that the observed differences in vascular markers are due to qualitative rather than quantitative differences in the raised serum NEFA. Since circulating TAG-rich lipoproteins have been shown to interact directly with the endothelium, the possibility that differences in the composition of TAG in these particles may be responsible for the observed effects on endothelial function should also be considered.

One mechanism linking fatty acids to vascular function is the potential effect on insulin secretion, as insulin is an important mediator of cardiovascular health ${ }^{(41)}$. Consumption of both fat loads caused a prompt increase in serum insulin peaking at $60 \mathrm{~min}$, with a concomitant increase in serum C-peptide. Both saturated and unsaturated NEFA have been shown to act directly on pancreatic $\beta$-cells to acutely increase insulin secretion in vitro; however, in vivo, the impact of NEFA on insulin depends on the prevailing glucose concentration, with a limited effect of NEFA at low glucose concentrations ${ }^{(42)}$. Although the glucose load in the drinks was minimal, a small increase in blood glucose concentration was observed at $30 \mathrm{~min}$ for all drinks, including the control, which suggests that the early insulin peak observed for the two fat-containing drinks reflects glucose-stimulated insulin secretion. Enhanced insulin secretion at $60 \mathrm{~min}$ observed for the fat-containing drinks, despite similar increases in glucose at $30 \mathrm{~min}$ for all three drinks, illustrates the potentiating effect of oral fatty acids on glucose-stimulated insulin secretion and influence of fat ingestion on glucose homeostasis ${ }^{(43)}$. It has long been known that oral fat is the most potent stimulus to GIP secretion. In vitro studies have shown that fatty acids can directly influence the release of GIP from the K-cells of the intestine, and that GIP binds to specific G-protein-linked receptors on the surface of the pancreatic $\beta$-cells to stimulate insulin secretion ${ }^{(42)}$. Although the overall GIP responses were similar for the two fats, there was a higher GIP concentration at $90 \mathrm{~min}$ for the MUFA- $v$. the SFA-rich fat load, which may have contributed to the second insulin response at $150 \mathrm{~min}$ for the MUFA fat load, which was not observed for the SFA fat load. The findings in the present study are consistent with those of Thomsen et $a l^{(20)}$, who reported a greater increase in GIP and GLP-1 after consumption of a mixed 
meal containing olive oil $v$. butter, as well as those of Brynes et al. ${ }^{(44)}$ who reported a trend towards greater GLP-1 levels after consumption of olive oil $v$. butter or maize oil. In contrast, Zampelas et $a l .{ }^{(45)}$ found no differences in GIP response after consumption of mixed meals rich in either SFA, $n-6$ PUFA or $n$-3 PUFA. We have no direct evidence to link the more adverse effect of a SFA- than a MUFA-rich fat load on arterial stiffness to the differences that we have observed in circulating incretin or insulin, but the protocol that we have used does add further support for an acute impact of fatty acid composition on the entero-insular axis.

Previous studies of the effect of dietary fat on markers of endothelial dysfunction have produced inconsistent findings. Nappo et al. ${ }^{(46)}$ found that consumption of a high-fat meal increased circulating sI-CAM levels, but Dekker et al. ${ }^{(47)}$ found no effect of a series of high-fat meals varying in their polyunsaturated:saturated ratio on either sI-CAM or soluble vascular adhesion molecule-1 levels. The present study found that acute elevation of NEFA during consumption of a SFA-rich but not of a MUFA-rich fat load resulted in modestly increased levels of SI-CAM, a circulating indicator of endothelial inflammation, which has been proposed as a useful marker of underlying coronary artery disease in healthy subjects $^{(48)}$. Although there are a number of mechanisms whereby elevation of NEFA may be linked with endothelial inflammation and dysfunction ${ }^{(32,48,49)}$, an increase in sI-CAM may indicate imbalance in insulin signalling in the endothelium since stimulation of the mitogen-activated protein kinase pathway, but not of the phosphoinositide 3-kinase pathway, results in an increase in the expression of cellular adhesion molecules, including sI-CAM. This may have a significant impact on endothelial function, as insulin signalling is a mediator of vascular tone through the generation of NO via the phosphoinositide 3-kinase pathway and the vasoconstrictor ET-1 via the mitogen-activated protein kinase pathway; this latter pathway can also lead to the generation of prothrombotic PAI- ${ }^{(41)}$. However, the present study found no difference in the response to the different test drinks of circulating markers of endothelium function (ET-1, NO $x$ and PAI-1) other than the modest increase that we have observed in sI-CAM. There is therefore insufficient evidence to conclude that the increase in arterial stiffness reported with the SFArich fat load is related to the impairment of insulin signalling pathways that regulate NO release or the expression of these proteins.

The protocol used in the present study achieved a successful threefold elevation of circulating NEFA, comparable with that found in the study of Beysen et al. ${ }^{(26)}$. Subjects in the present study had higher fasting levels of NEFA (average 667 $\mu \mathrm{mol} / \mathrm{l}$ ) compared with the group of Beysen et al. (260-400 $\mu \mathrm{mol} / \mathrm{l})$, which may have contributed to the higher absolute NEFA values that we observed following heparin administration. A limitation of our sample processing meant that up to $30 \mathrm{~min}$ could have elapsed before samples were stored at $4^{\circ} \mathrm{C}$; this could have resulted in a degree of in vitro lipolysis in the post-heparin samples and may have contributed to the higher values that we have reported here.
In conclusion, the main finding of the present study is that an acute elevation of serum NEFA following consumption of a SFA-rich drink has an adverse impact on arterial stiffness and sI-CAM levels. The study also confirms that ingestion of a fat load leads to sustained increases in the incretin hormone GIP, with some evidence for a more marked effect of MUFA than for SFA. It is not possible to determine whether the effects of different fats on vascular tone and endothelial inflammation are related to differential effects on incretin or insulin secretion and/or action at the endothelium. However, given the known effects of NEFA elevation on other insulin-sensitive tissues such as skeletal muscle and adipose tissue, further elucidation of the effects of fat type on the sensitivity of the endothelium to insulin is warranted.

\section{Acknowledgements}

The present study was supported by the BBSRC (Swindon, Wiltshire, UK; BB/E0221816/1) Unilever PLC (Colworth Park, Sharnbrook, Bedfordshire, UK) and FRST (Wellington, New Zealand). The fats were kindly donated by Aarhuskarlshman Limited (Hull, UK). The authors wish to thank our volunteers for their participation, and Matthew Barton, Nadine Becker, Agnieszka Przemska, Dafni Vasilopoulou and Alice Turner for their help during the study days. We are also grateful to Duncan Talbot for his expertise in insulin measurement, and Sue Todd for her statistical advice. The authors had no conflicts of interest. The authors' contributions are as follows: A. K. T., K. J. N. and J. W. conducted the study; K. J. N. performed statistical analyses and drafted the manuscript; all authors contributed to the design and analysis of the study as well as to manuscript preparation.

\section{References}

1. Charles MA, Eschwege E, Thibult N, et al. (1997) The role of non-esterified fatty acids in the deterioration of glucose tolerance in Caucasian subjects: results of the Paris Prospective Study. Diabetologia 40, 1101-1106.

2. Wilding JP (2007) The importance of free fatty acids in the development of type 2 diabetes. Diabet Med 24, 934-945.

3. Belfort R, Mandarino L, Kashyap S, et al. (2005) Doseresponse effect of elevated plasma free fatty acid on insulin signaling. Diabetes 54, 1640-1648.

4. Steinberg HO, Tarshoby M, Monestel R, et al. (1997) Elevated circulating free fatty acid levels impair endotheliumdependent vasodilation. J Clin Invest 100, 1230-1239.

5. Steinberg HO, Chaker H, Leaming R, et al. (1996) Obesity/ insulin resistance is associated with endothelial dysfunction. Implications for the syndrome of insulin resistance. $J$ Clin Invest 97, 2601-2610.

6. Hogikyan RV, Galecki AT, Pitt B, et al. (1998) Specific impairment of endothelium-dependent vasodilation in subjects with type 2 diabetes independent of obesity. J Clin Endocrinol Metab 83, 1946-1952.

7. Miller M, Beach V, Sorkin JD, et al. (2009) Comparative effects of three popular diets on lipids, endothelial function, and C-reactive protein during weight maintenance. J Am Diet Assoc 109, 713-717.

8. Steer P, Vessby B \& Lind L (2003) Endothelial vasodilatory function is related to the proportions of saturated fatty 
acids and alpha-linolenic acid in young men, but not in women. Eur J Clin Invest 33, 390-396.

9. Sarabi M, Vessby B, Millgard J, et al. (2001) Endotheliumdependent vasodilation is related to the fatty acid composition of serum lipids in healthy subjects. Atherosclerosis 156, 349-355.

10. Keogh JB, Grieger JA, Noakes M, et al. (2005) Flow-mediated dilatation is impaired by a high-saturated fat diet but not by a high-carbohydrate diet. Arterioscler Thromb Vasc Biol 25, 1274-1279.

11. Lind L, Sodergren E, Gustafsson IB, et al. (2002) The types of circulating fatty acids influence vascular reactivity. Lipids $\mathbf{3 7}$, 1141-1145.

12. Khan F, Elherik K, Bolton-Smith C, et al. (2003) The effects of dietary fatty acid supplementation on endothelial function and vascular tone in healthy subjects. Cardiovasc Res 59, 955-962.

13. Rueda-Clausen CF, Silva FA, Lindarte MA, et al. (2007) Olive, soybean and palm oils intake have a similar acute detrimental effect over the endothelial function in healthy young subjects. Nutr Metab Cardiovasc Dis 17, 50-57.

14. Raitakari OT, Lai N, Griffiths K, et al. (2000) Enhanced peripheral vasodilation in humans after a fatty meal. $J \mathrm{Am}$ Coll Cardiol 36, 417-422.

15. Berry SE, Tucker S, Banerji R, et al. (2008) Impaired postprandial endothelial function depends on the type of fat consumed by healthy men. J Nutr 138, 1910-1914.

16. Galgani JE, Uauy RD, Aguirre CA, et al. (2008) Effect of the dietary fat quality on insulin sensitivity. Br J Nutr 100, 471-479.

17. Vessby B, Unsitupa M, Hermansen K, et al. (2001) Substituting dietary saturated for monounsaturated fat impairs insulin sensitivity in healthy men and women: The KANWU Study. Diabetologia 44, 312-319.

18. Wang L, Folsom AR, Zheng ZJ, et al. (2003) Plasma fatty acid composition and incidence of diabetes in middle-aged adults: the Atherosclerosis Risk in Communities (ARIC) Study. Am J Clin Nutr 78, 91-98.

19. Nystrom T, Gutniak MK, Zhang Q, et al. (2004) Effects of glucagon-like peptide- 1 on endothelial function in type 2 diabetes patients with stable coronary artery disease. $A m \mathrm{~J}$ Physiol Endocrinol Metab 287, E1209-E1215.

20. Thomsen C, Rasmussen O, Lousen T, et al. (1999) Differential effects of saturated and monounsaturated fatty acids on postprandial lipemia and incretin responses in healthy subjects. Am J Clin Nutr 69, 1135-1143.

21. Robertson MD, Jackson KG, Fielding BA, et al. (2002) Acute ingestion of a meal rich in $n-3$ polyunsaturated fatty acids results in rapid gastric emptying in humans. Am J Clin Nutr 76, 232-238.

22. Jackson KG, Zampelas A, Knapper JM, et al. (2000) Differences in glucose-dependent insulinotrophic polypeptide hormone and hepatic lipase in subjects of southern and northern Europe: implications for postprandial lipemia. $\mathrm{Am}$ J Clin Nutr 71, 13-20.

23. Casey DP, Beck DT \& Braith RW (2007) Systemic plasma levels of nitrite/nitrate $\left(\mathrm{NO}_{x}\right)$ reflect brachial flow-mediated dilation responses in young men and women. Clin Exp Pharmacol Physiol 34, 1291-1293.

24. Wang XL, Zhang L, Youker K, et al. (2006) Free fatty acids inhibit insulin signaling-stimulated endothelial nitric oxide synthase activation through upregulating PTEN or inhibiting Akt kinase. Diabetes 55, 2301-2310.

25. Xiao-Yun X, Zhuo-Xiong C, Min-Xiang L, et al. (2009) Ceramide mediates inhibition of the AKT/eNOS signaling pathway by palmitate in human vascular endothelial cells. Med Sci Monit 15, BR254-BR261.

26. Beysen C, Belcher AK, Karpe F, et al. (2003) Novel experimental protocol to increase specific plasma nonesterified fatty acids in humans. Am J Physiol Endocrinol Metab 284, E18-E24.

27. Beysen C, Karpe F, Fielding BA, et al. (2002) Interaction between specific fatty acids, GLP-1 and insulin secretion in humans. Diabetologia 45, 1533-1541.

28. Wykretowicz A, Gerstenberger P, Guzik P, et al. (2009) Arterial stiffness in relation to subclinical atherosclerosis. Eur J Clin Invest 39, 11-16.

29. Kew S, Mesa MD, Tricon S, et al. (2004) Effects of oils rich in eicosapentaenoic and docosahexaenoic acids on immune cell composition and function in healthy humans. Am J Clin Nutr 79, 674-681.

30. Steer P, Basu S, Lithell H, et al. (2003) Acute elevations of medium- and long-chain fatty acid have different impacts on endothelium-dependent vasodilation in humans. Lipids 38, 15-19.

31. Kearney MT, Chowienczyk PJ, Brett SE, et al. (2002) Acute haemodynamic effects of lipolysis-induced increase of free fatty acids in healthy men. Clin Sci (Lond) 102, 495-500.

32. Tripathy D, Mohanty P, Dhindsa S, et al. (2003) Elevation of free fatty acids induces inflammation and impairs vascular reactivity in healthy subjects. Diabetes 52, 2882-2887.

33. Stepniakowski KT, Lu G, Davda RK, et al. (1997) Fatty acids augment endothelium-dependent dilation in hand veins by a cyclooxygenase-dependent mechanism. Hypertension 30, $1634-1639$.

34. Pleiner J, Schaller G, Mittermayer F, et al. (2002) FFA-induced endothelial dysfunction can be corrected by vitamin C. J Clin Endocrinol Metab 87, 2913-2917.

35. de Jongh RT, Serne EH, Ijzerman RG, et al. (2004) Free fatty acid levels modulate microvascular function: relevance for obesity-associated insulin resistance, hypertension, and microangiopathy. Diabetes 53, 2873-2882.

36. Lind L, Fugmann A, Branth S, et al. (2000) The impairment in endothelial function induced by non-esterified fatty acids can be reversed by insulin. Clin Sci (Lond) 99, 169-174.

37. Jackson KG, Armah CK \& Minihane AM (2007) Meal fatty acids and postprandial vascular reactivity. Biochem SOC Trans 35, 451-453.

38. Hunter JE, Zhang J \& Kris-Etherton PM (2010) Cardiovascular disease risk of dietary stearic acid compared with trans, other saturated, and unsaturated fatty acids: a systematic review. Am J Clin Nutr 91, 46-63.

39. Marchesi S, Lupattelli G, Schillaci G, et al. (2000) Impaired flow-mediated vasoactivity during post-prandial phase in young healthy men. Atherosclerosis 153, 397-402.

40. Bae JH, Schwemmer M, Lee IK, et al. (2003) Postprandial hypertriglyceridemia-induced endothelial dysfunction in healthy subjects is independent of lipid oxidation. Int $J$ Cardiol 87, 259-267.

41. Muniyappa R, Montagnani M, Koh KK, et al. (2007) Cardiovascular actions of insulin. Endocr Rev 28, 463-491.

42. Itoh Y, Kawamata Y, Harada M, et al. (2003) Free fatty acids regulate insulin secretion from pancreatic beta cells through GPR40. Nature 422, 173-176.

43. Reimann F (2010) Molecular mechanisms underlying nutrient detection by incretin-secreting cells. Int Dairy $J \mathbf{2 0}$, 236-242.

44. Brynes AE, Frost GS, Edwards CM, et al. (1998) Plasma glucagon-like peptide-1 (7-36) amide (GLP-1) response to liquid phase, solid phase, and meals of differing lipid composition. Nutrition 14, 433-436. 
45. Zampelas A, Murphy M, Morgan LM, et al. (1994) Postprandial lipoprotein lipase, insulin and gastric inhibitory polypeptide responses to test meals of different fatty acid composition: comparison of saturated, $n-6$ and $n-3$ polyunsaturated fatty acids. Eur J Clin Nutr 48, 849-858.

46. Nappo F, Esposito K, Cioffi M, et al. (2002) Postprandial endothelial activation in healthy subjects and in type 2 diabetic patients: role of fat and carbohydrate meals. $J \mathrm{Am}$ Coll Cardiol 39, 1145-1150.
47. Dekker MJ, Wright AJ, Mazurak VC, et al. (2008) Fasting triacylglycerol status, but not polyunsaturated/saturated fatty acid ratio, influences the postprandial response to a series of oral fat tolerance tests. I Nutr Biochem 20, 694-704.

48. Blankenberg S, Barbaux S \& Tiret L (2003) Adhesion molecules and atherosclerosis. Atherosclerosis 170, 191-203.

49. Calder PC (2002) Dietary modification of inflammation with lipids. Proc Nutr SOC 61, 345-358. 